\title{
Stable isotopic evidence for microbial ethane in Lake Baikal gas hydrates
}

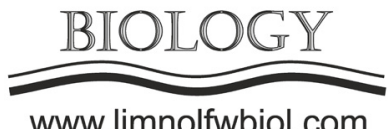

www.limnolfwbiol.com

\author{
Hachikubo A. ${ }^{1 *}$, Kimura H. ${ }^{1}$, Kamata R. ${ }^{1}$, Sakagami H. ${ }^{1}$, Minami H. ${ }^{1}$, \\ Yamashita S. ${ }^{1}$, Khlystov O. ${ }^{2}$, Kalmychkov G. ${ }^{3}$, De Batist M. ${ }^{4}$ \\ ${ }^{1}$ Kitami Institute of Technology, 165 Koen-cho, Kitami 090-8507, Japan \\ ${ }^{2}$ Limnological Institute, Siberian Branch of the Russian Academy of Sciences, Ulan-Batorskaya Str., 3, Irkutsk 664033, Russia \\ ${ }^{3}$ Vinogradov Institute of Geochemistry, Siberian Branch of the Russian Academy of Sciences, Favorsky Str., 1-a, Irkutsk 664033, \\ Russia \\ ${ }^{4}$ Renard Centre of Marine Geology, Ghent University, Krijgslaan 281s8, 9000, Ghent, Belgium
}

\begin{abstract}
In the framework of Multi-phase Gas Hydrate Project, near-surface gas hydrates were retrieved from the southern and central Baikal basins. In this report, we summarize characteristics of hydrate-bound hydrocarbons retrieved at Lake Baikal and focus on hydrate-bound ethane and its gas origin. The information of molecular composition of volatile hydrocarbons and their stable isotopes showed that the origin of hydrate-bound gas distributes in the fields of microbial, thermogenic, and their mixed-gas. As for stable isotope of ethane, $\delta^{13} \mathrm{C}$ and $\delta \mathrm{D}$ distribute from $-69 \%$ to $-23 \%$ V-PDB, and from $-313 \%$ to $-185 \%$ V-SMOW, respectively. Light ethane in $\delta^{13} \mathrm{C}$ also depleted in $\delta \mathrm{D}$, indicating that the isotopically light ethane depleted in ${ }^{13} \mathrm{C}$ and $\mathrm{D}$ is generated by a microbial process, and the origin of hydrogen in ethane molecules is thought to be lake water, same as methane.
\end{abstract}

Keywords: gas hydrate, ethane, microbial gas, isotopic fractionation, gas origin

\section{Introduction}

Natural gas hydrate exists in sublacustrine sediments of Lake Baikal. In the framework of Multiphase Gas Hydrate Project (MHP, 2009-2019) between Russia, Belgium, and Japan, has revealed characteristics of near-surface gas hydrates retrieved from the southern and central Baikal basins. We measured molecular and isotopic compositions of hydrate-bound gases retrieved from eight places and discussed their gas origin (Hachikubo et al., 2010). Recently, we found six new places (Zelen MV, Zelen Seep, Katko MV, BelKamen MV, Melky Seep, and Kukuy K-17 MV) during the cruise of VER19-03 in 2019. The total number of places where near-surface gas hydrates were retrieved at Lake Baikal reached 60 . In this study, we summarize characteristics of hydrate-bound gases retrieved from all the sites since 2005, and focus on hydrate-bound ethane and its gas origin.

\section{Material and methods}

Hydrate-bearing sediment cores were recovered using R/V "G.Yu. Vereshchagin" equipped with a gravity corer. Gas hydrate samples were retrieved from splitted sediment cores. Gas sampling was conducted for each layer of gas hydrate in the sediment cores including a core-catcher. In the case of massive hydrate nodules, several samples were collected along to the depth. Hydrate-bound gas were collected by the water displacement method using a funnel and plastic bucket filled with water, and stored in glass vials with butylrubber septum. No air contamination was expected, but small amount of water still remained. To avoid microbial activity in the vials, $10 \mu \mathrm{L}$ of benzalkonium chloride (50 wt $\%$ aqueous solution) was introduced into them using a syringe (Waseda and Iwano, 2007).

The molecular compositions of the hydrocarbons (from methane to pentane) were determined using a gas chromatograph (GC2014, Shimadzu) equipped with a packed column (Shimadzu Sunpak-S), along with a thermal conductivity detector and flame ionization detector for detecting high and low concentrations of hydrocarbons, respectively. The two detectors were connected in series. The stable carbon and hydrogen isotopic ratios of the hydrocarbons were measured using a CF-IRMS (Delta V, Thermo Fisher Scientific) coupled with a gas chromatograph (Trace GC Ultra, Thermo Fisher Scientific). The gas chromatograph was equipped with a Carboxen-1006 PLOT capillary column. Stable isotope compositions are reported as $\delta$ values (\%), and $\delta^{13} \mathrm{C}$ and $\delta \mathrm{D}$ are given with reference to the V-PDB and V-SMOW standards, respectively. 


\section{Results and discussion}

The information of molecular composition of volatile hydrocarbons and their stable isotopes showed that the origin of hydrate-bound gas distributes in the fields of microbial, thermogenic, and their mixedgas. Ethane $\delta^{13} \mathrm{C}$ distributed from $-69 \%$ (Solzan, gas hydrate mound at the southern Baikal basin) to $-23 \%$ (Kukuy K-P, pockmark at the central Baikal basin). The relation between methane $\delta^{13} \mathrm{C}$ and ethane $\delta^{13} \mathrm{C}$ based on Milkov's diagram (Milkov, 2005) showed "L shape", suggesting that hydrate-bound ethane in Lake Baikal is composed of ethane-rich thermogenic gas and ethanedepleted microbial gas. In the case of mixed-gas, methane $\delta^{13} \mathrm{C}$ decreased whereas ethane $\delta^{13} \mathrm{C}$ kept the value of thermogenic gas, because the concentration of ethane in microbial gas was low.

According to the $\delta^{13} \mathrm{C}-\delta \mathrm{D}$ diagram for methane (Whiticar et al., 1986), methane $\delta \mathrm{D}$ provides information on methyl-type fermentation or $\mathrm{CO}_{2}$ reduction in the microbial origin; however, this interpretation is still in controversy. Methane $\delta \mathrm{D}$ in Lake Baikal gas hydrates distributed from $-330 \%$ to $-270 \%$, whereas those in marine gas hydrates distributed from $-200 \%$ to $-140 \%$ o (Milkov, 2005). Therefore, methane $\delta \mathrm{D}$ of Lake Baikal gas hydrates seems to be around $130 \%$ smaller than that of marine gas hydrates. Matveeva et al. (2003) reported that $\delta \mathrm{D}$ of the lake bottom water was about $-133 \%$. It is concluded that methane $\delta \mathrm{D}$ of hydratebound methane derives from $\delta \mathrm{D}$ of water.

Hachikubo et al. (2010) proposed a diagram, relation between $\delta^{13} \mathrm{C}$ and $\delta \mathrm{D}$ of ethane, similar to the Whiticar diagram (Whiticar et al., 1986). The diagram showed that light ethane in $\delta^{13} \mathrm{C}$ also depleted in $\delta \mathrm{D}$; hydrate-bound thermogenic ethane is plotted around $-25 \%$ in $\delta^{13} \mathrm{C}$ and $-210 \%$ in $\delta \mathrm{D}$, whereas those of microbial ethane is plotted around $-60 \%$ in $\delta^{13} \mathrm{C}$ and $-280 \%$ in $\delta \mathrm{D}$. These results indicate that the light ethane depleted in ${ }^{13} \mathrm{C}$ and $\mathrm{D}$ is generated by a microbial process, and the origin of hydrogen in ethane molecules is likely to be lake water, same as methane.

\section{Conclusions}

We found light ethane in the hydrate-bound gases both in $\delta^{13} \mathrm{C}$ and $\delta \mathrm{D}$, suggesting that Lake Baikal gas hydrates enclathrate microbial ethane with methane. To confirm microbial ethane, incubation experiments of ethanogenesis using lake-bottom sediments will be needed.

\section{Acknowledgments}

We appreciate the assistance of the shipboard crews of RV "G.Yu. Vereshchagin" during the Lake Baikal expeditions. This work was supported by funding agencies in Japan (Japan Society for the Promotion of Science KAKENHI 26303021, 16H05760 and $17 \mathrm{H03300}$ and the presidential grant of the Kitami Institute of Technology).

\section{References}

Hachikubo A., Khlystov O., Krylov A. et al. 2010. Molecular and isotopic characteristics of gas hydrate-bound hydrocarbons in southern and central Lake Baikal. Geo-Marine Letters 30: 321-329. DOI: 10.1007/s00367-010-0203-1

Matveeva T.V., Mazurenko L.L., Soloviev V.A. et al. 2003. Gas hydrate accumulation in the subsurface sediments of Lake Baikal (Eastern Siberia). Geo-Marine Letters 23(3/4): 289-299. DOI: 10.1007/s00367-003-0144-7

Milkov A.V. 2005. Molecular and stable isotope compositions of natural gas hydrates: a revised global dataset and basic interpretations in the context of geological settings. Organic Geochemistry 36: 681-702. DOI: 10.1016/j. orggeochem.2005.01.010

Waseda A., Iwano H. 2007. Reservoir evaluation using carbon isotope composition of gas. Journal of the Japanese Association for Petroleum Technology 72(6): 585-593. DOI: 10.3720/japt.72.585 (in Japanese)

Whiticar M.J., Faber E., Schoell M. 1986. Biogenic methane formation in marine and freshwater environments: $\mathrm{CO}_{2}$ reduction $v s$. acetate fermentation-isotope evidence. Geochimica et Cosmochimica Acta 50: 693-709. DOI: 10.1016/0016-7037(86)90346-7 